\title{
Répteis que um dia dominaram os mares
}

\author{
ReptILES THAT ONCE DOMINATED tHE SEAS
}

\author{
Geraldo Norberto Chaves Sgarbi ${ }^{1}$, Jonathas Bittencourt ${ }^{2}$, Thiago da Silva Marinho ${ }^{3}$ \\ 1-IGC/UFMG, Av. Antônio Carlos, 6627, Pampulha, Belo Horizonte-MG, gncsgarbi@gmail.com \\ 2-IGC/UFMG, Av. Antônio Carlos, 6627, Pampulha, Belo Horizonte-MG, bittencourt.paleo@gmail.com \\ 3-ICENE/UFTM, Av. Dr. Randolfo Borges Jr. 1400, Univerdecidade, Uberaba-MG, tsmarinho@gmail.com
}

\begin{abstract}
The Mesozoic Era is a fascinating period in the evolution of life on Earth. Several animal taxa with representatives in present-day fauna, such as amphibians, mammals, birds, turtles, lizards, snakes and crocodiles, originated and diversified in this Era. Non-avian dinosaurs and pterosaurs also occur within this geological time span, making it known as the Age of Reptiles. Less known to the public is the fact that oceans and seas were also ruled by extinct reptiles, including ichthyosaurs, plesiosaurs and mosasaurs. Their singular anatomy and complete demise at the end of Mesozoic continue to fascinate, ever since their discovery starting in the $18^{\text {th }}$ century. This work provides information to teachers so as to support basic education about the main groups of marine reptiles of the Mesozoic. We discuss aspects concerning anatomy, lifestyle, evolution and the implications of their discovery for the history of science.
\end{abstract}

Manuscrito:

Recebido: 25/04/2015

Corrigido: $15 / 12 / 2015$

Aceito: 01/02/2016

Citation: Sgarbi G.N.C., Bittencourt J., Marinho T.S. 2016. Répteis que um dia dominaram os mares. Terræ Didatica, 12(1):69-77. < http:// www.ige.unicamp.br/terraedidatica/>.

Keywords:Paleontology, extinction, fossil reptiles, aquatic paleoenvironments.
A primeira ideia que, em geral, vem à mente das pessoas quando se pronuncia a palavra "dinossauro" é a de gigantescos répteis, ou mais comumente "lagartos", que caminharam sobre a Terra em um passado remoto, quando o homem ainda estava em sua idade da pedra. São conceitos equivocados, uma vez que nem todos os dinossauros eram gigantes, não eram "lagartos" e se extinguiram (exceto as aves) quase 65 milhões de anos (Ma) antes da origem do homem. Também é comum associar qualquer animal pré-histórico com os dinossauros, mesmo que, na realidade, seja de uma linhagem evolutiva muito distinta de répteis, ou ainda, parente mais próximo dos mamíferos (por exemplo, preguiças terrícolas gigantes).

Este trabalho tem como objetivo discutir os principais grupos de répteis que dominaram os ambientes marinhos durante a Era Mesozoica (250 a $65 \mathrm{Ma}$ ) que não são classificados como dinossauros, embora sejam seus contemporâneos. Há diversos grupos de répteis marinhos no Mesozoico, incluindo serpentes, tartarugas, crocodiliformes (grupo que inclui os jacarés atuais), mas será dada ênfase aos principais animais que não fazem parte de grupos com representantes atuais, como os ictiossauros, plesiossauros e mosassauros.

À semelhança de trabalhos prévios, que abordaram a fauna de outros grupos de répteis do Mesozoico (Sgarbi 1999, 2003), este artigo traz informações sobre a anatomia, modo de vida e evolução desses animais, além de aspectos históricos sobre sua descoberta. Espera-se que se torne uma ferramenta útil, atualizada e tecnicamente correta, para docentes do ensino básico que busquem atividades extras no ensino de ciências.

\section{Ictiossauros: os répteis-peixes do Mesozoico}

Os ictiossauros mais conhecidos são, superficialmente, semelhantes aos golfinhos (Fig. 1). Seu corpo reflete uma série de adaptações que permitem uma maior hidrodinâmica, como a forma em fuso (semicírculo esticado nas pontas), parte anterior do crânio alongada, uma nadadeira dorsal 
(não em todas as espécies), duas peitorais, duas pélvicas e uma caudal, além da posição das narinas no topo do crânio, que permite a respiração na superfície (Benton 2006). Esses animais tinham pulmões, como os demais répteis, e se utilizavam do ar atmosférico para respirar.

Mas as diferenças entre esses grupos são muito marcantes, como a presença de nadadeiras pélvicas nos ictiossauros, uma característica que está associada com a presença dos membros posteriores, ao contrario dos mamíferos aquáticos modernos, que as perderam ao longo de sua evolução (Carroll 1988). Além disso, nos ictiossauros a nadadeira caudal movimenta lateralmente para propulsão (Motani et al. 1996), como nos peixes, e não dorso-ventralmente, como nas baleias e golfinhos.

Quando se analisa o esqueleto, as diferenças são ainda mais visíveis. Os ossos que compõem o crânio dos ictiossauros são mais parecidos com os dos répteis, como os jacarés e lagartos, e as patas são modificadas por uma característica chamada de hiperfalangia, na qual há um aumento no número de falanges (os ossos dos dedos) e polidactilia (aumento do número de dígitos) (Carroll 1988, Pough et al. 1999). Essas características conferem à nadadeira uma importante função de direcionamento durante a locomoção.

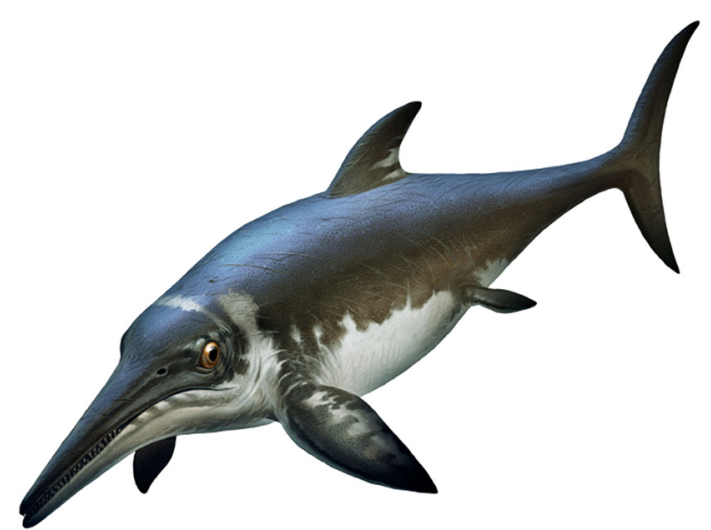

Figura 1. O ictiossauro Platypterygius, que podia atingir cerca de 7 metros de comprimento. Imagem: Xing Lida.

Fósseis identificados como ictiossauros permitem inferir que esses animais viveram desde o início do Triássico $(250 \mathrm{Ma})$ até meados do Cretáceo (90 Ma) (Benton 2006). No entanto, a maior diversidade desse grupo foi encontrada no Jurássico (de 200 a $145 \mathrm{Ma}$ ). Alguns aspectos de sua biologia são conhecidos, como a dieta baseada em moluscos cefalópodes (parecidos com as lulas atuais) e peixes, embora conteúdo estomacal de alguns fósseis com tartarugas e aves também tenha sido encontrado (Kear et al. 2003). Alguns dados baseados em isótopos estáveis de oxigênio, que ficam preservados nos fósseis, mostram que esses animais podiam manter a temperatura corporal constante (Bernard et al. 2010), semelhante a aves e mamíferos - processo conhecido por homeotermia.

$\mathrm{O}$ tamanho dos indivíduos poderia variar de cerca de 1 a 15 metros de comprimento (Vitt \& Caldwell 2014), embora haja registros de que pudessem atingir 20 metros de comprimento (Nicholls e Manabe 2004). A visão deveria ser um componente importante dos sentidos, já que os ictiossauros têm os maiores globos oculares dentre os animais. Um gênero encontrado na Europa e América do Norte, chamado oftalmossauro (Ophthalmosau$r u s)$, tinha uma órbita ocular, isto é, a cavidade no crânio onde se insere o olho, de 23 centímetros, para um comprimento total do corpo de 4 metros (Motani s/d). A baleia azul, que pode chegar a 30 metros de comprimento, tem uma órbita de 15 centímetros. Comparações com animais marinhos atuais com respiração aérea permitem inferir que o oftalmossauro poderia submergir por no mínimo 20 minutos, a uma profundidade de 600 a $1.500 \mathrm{~m}$ (Motani et al. 1999).

Fósseis de ictiossauros muito bem preservados nos calcários laminados de Holzmaden, no sul da Alemanha (Organ et al. 2009), mostram que esses animais seriam vivíparos, processo no qual as fêmeas dão à luz juvenis sem a formação de ovo (Fig. 2). Alguns fósseis mostram fêmeas com um ou dois filhotes em gestação, mas esse número poderia chegar a onze (Böttcher 1990). Semelhante aos golfinhos e baleias, os filhotes nasciam com a cauda saindo primeiro do corpo da mãe, depois a cabeça, evitando o afogamento. No entanto, um fóssil de ictiossauro do início da Era Mesozoica mostra uma fêmea dando à luz um filhote com a cabeça saindo primeiramente do corpo (Motani et al. 2014), sugerindo que, no início da evolução do grupo, esse processo se dava em ambiente terrestre.

A origem dos ictiossauros ainda é um dos grandes enigmas da paleontologia. Sabe-se que, como muitos outros répteis da era Mesozoica, incluindo os pterossauros, plesiossauros e mosassauros, os ictiossauros não são dinossauros. Estudos de relação de parentesco, ou filogenia (Fig. 3), sugerem que 


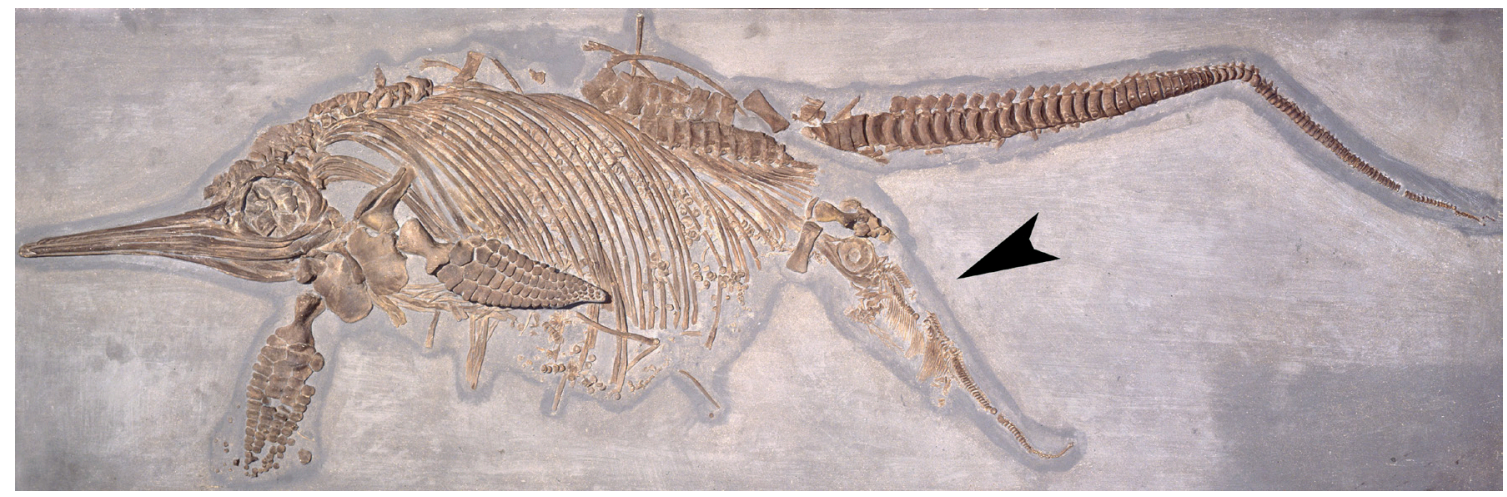

Figura 2. O ictiossauro Stenopterygius (c. 2 m), preservado junto com filhote (indicado pela seta). Imagem: Organ et al. (2009). (c) The Natural History Museum, Londres.

os ictiossauros se adaptaram ao ambiente aquático a partir de ancestrais terrestres diápsidos (Benton 2006). Estes formam um grande grupo de répteis que também inclui os lagartos e serpentes (ou lepidossauros) e os crocodilos e aves (ou arcossauros). A hipótese ganhou um grande reforço com a descoberta do parente mais próximo dos ictiossauros, Cartorhynchus, proveniente de rochas dos Triássico Inferior da China (Motani et al. 2015). Com características que indicam um hábito anfíbio de vida, a nova espécie fornece evidências de que estes animais estão de fato relacionados aos répteis diápsidos.

\section{Plesiossauros: dragões-do-mar e o monstro do lago Ness}

Os plesiossauros são animais únicos, dificilmente comparáveis aos grupos modernos de répteis, e sem análogos na fauna marinha atual. $\mathrm{O}$ nome do grupo deriva do grego e significa "quase réptil" (De la Beche \& Conybeare 1821), devido à sua forma estranha quando comparada aos demais répteis. Seu corpo, que poderia variar de 2 a 15 metros, tinha uma cauda relativamente curta. Dependendo do grupo, o pescoço também era curto, mas com a cabeça comprida (os pliossauroides),

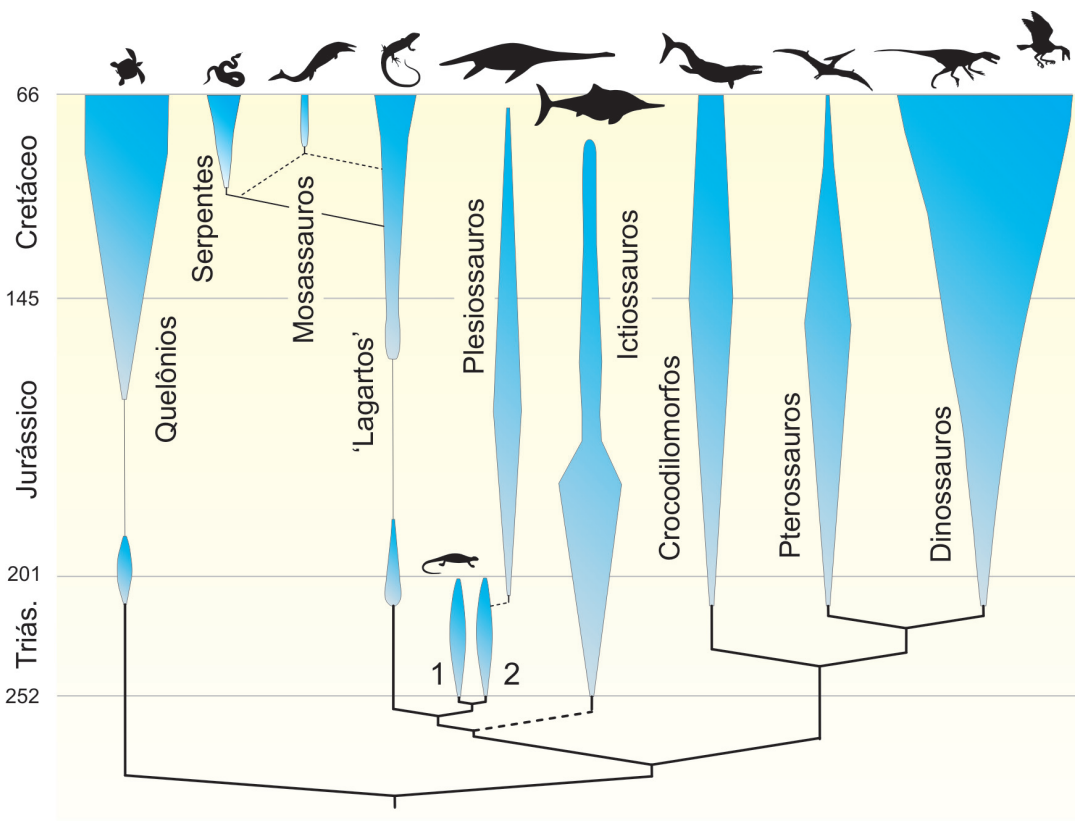

Figura 3. Relações de parentesco (filogenia) dos principais grupos de répteis. A espessura das linhagens indica diversidade durante o Mesozoico. As linhas tracejadas indicam dúvidas quanto ao parentesco evolutivo. Triás.: Triássico; 1 : Placodontes; 2: Notossauros. Modificado de Benton (2006). Algumas silhuetas redesenhadas a partir de Motani (2009). ou o pescoço era longo, devido ao aumento de vértebras cervicais, e a cabeça, curta (os plesiossauroides) (O'Keefe 2002, Benton 2006). Os plesiossauros da família dos elasmossaurídeos (Fig. 4) podiam ter 71 vértebras cervicais (Sachs 2005), enquanto a maioria dos dinossauros tem dez vértebras.

A construção corporal é tão estranha que, quando foi descoberto um dos maiores plesiossauros, o elasmossauro, o importante paleontólogo norte-americano Edward Cope reconstruiu erroneamente o animal com a cabeça na extremidade da cauda (Psihoyos 1994). Na época, a segunda metade de século XIX, os animais até então 


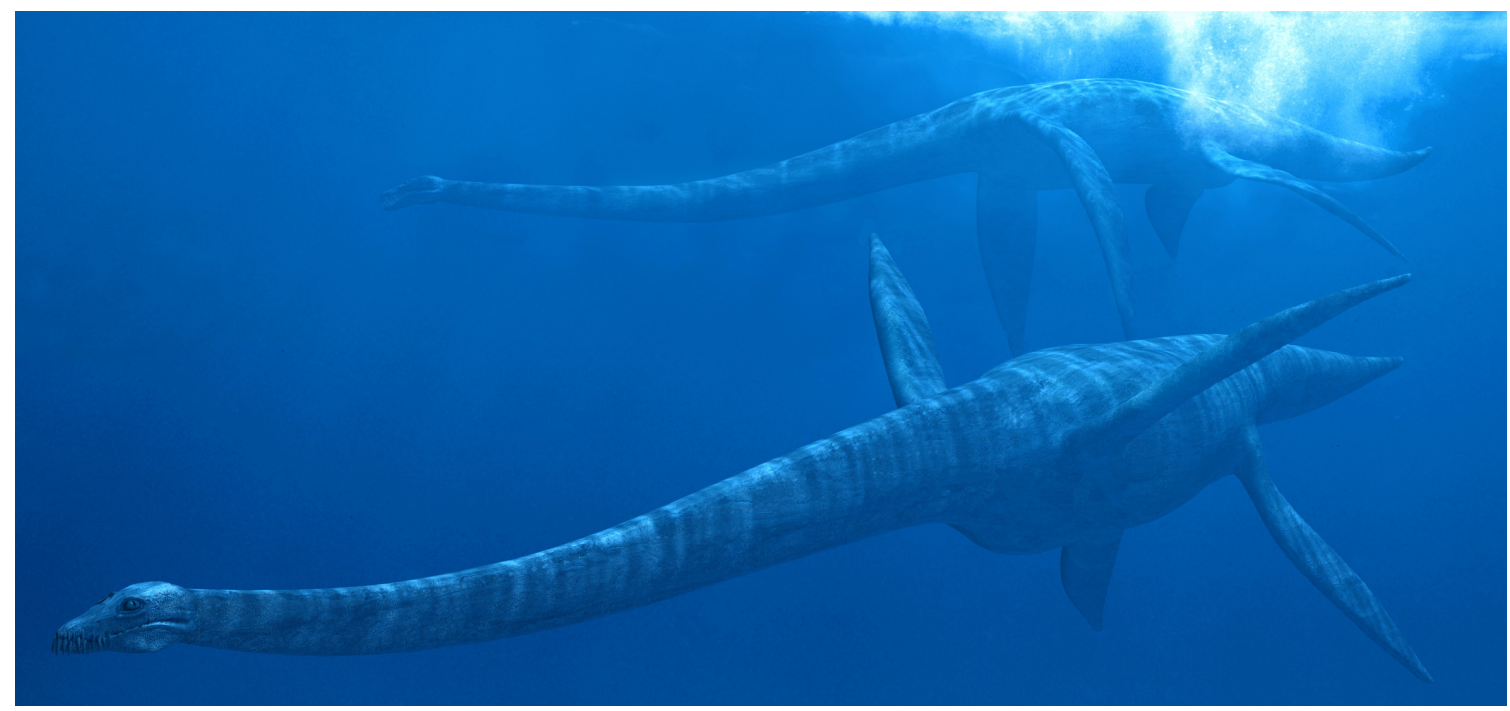

Figura 4. O plesiossauro Elasmosaurus (c. 14 m). Imagem: Frank DeNota.

conhecidos tinham, em geral, a cauda longa e o pescoço curto. Mesmo com poucos parâmetros de comparação, a descoberta do erro ainda em 1870 gerou imenso constrangimento pessoal para Cope (Psihoyos 1994).

Diversas adaptações para a locomoção aquática são conhecidas nos plesiossauros: corpo alongado, hiperfalangia, nadadeiras em forma de remo, narinas externas no topo do crânio, e a parte ventral do tronco encerrada por cinturas alongadas e a gastrália (ou "costelas" abdominais) (Carroll 1988, Pough et al. 1999).

Aspectos da biologia dos plesiossauros são conhecidos diretamente pelos fósseis ou inferidos a partir da anatomia. Plesiossauros têm dentes longos e afiados, expandindo-se para fora da zona de oclusão da mandíbula e maxila, formando uma cesta que poderia ser usada para aprisionar peixes e moluscos (Benton 2006). Vários plesiossauros foram encontrados associados a gastrólitos (Schmeisser \& Gillette 2009), que são seixos ingeridos pelo animal durante o forrageio, facilitando a digestão de material rígido (como conchas), e que podem ser preservados junto com o esqueleto no processo de fossilização. A ingestão de seixos também ocorre nas aves atuais, e esse material é chamado de pedras de moela.

Em termos fisiológicos, os plesiossauros eram possivelmente homeotérmicos, como os ictiossauros (Bernard et al. 2010). Com respeito à biomecânica, pesquisas recentes têm mostrado que as espécies de pescoço relativamente curto seriam mais velozes, e usavam uma estratégia de perseguição para caça, enquanto que os de pescoço longo seriam mais lentos, e por isso caçadores de emboscada (O'Keefe 2001).

Quanto à reprodução, o fóssil de uma fêmea do gênero Polycotylus com restos de um embrião na cavidade abdominal (Fig. 5) mostra que, como os ictiossauros, os plesiossauros eram vivíparos (O'Keefe \& Chiappe 2011). O espécime sugere que a prole era de poucos filhotes, mas a raridade desse tipo de achado não permite muitas inferências sobre a reprodução do grupo.

A descoberta da primeira espécie de plesiossauros, justamente o do gênero Plesiosaurus, tem uma importância muito grande para a história da Paleontologia. Os fósseis que serviram de base para a descrição da espécie, e o seu primeiro esqueleto completo alguns anos depois, foram descobertos pela naturalista inglesa Mary Anning na década de 20 dos anos 1800 em rochas do Jurássico Inferior, em Dorset, Inglaterra (Torrens 1995). Ela foi uma pioneira na prospecção dos fósseis que muito ajudaram na consolidação do interesse púbico pela Paleontologia e da teoria da evolução no fim do século XIX e início do XX (Torrens 1995, Turner 2010).

A origem dos plesiossauros, assim como dos ictiossauros, é um tema polêmico na paleontologia de vertebrados. Seus parentes mais próximos são também adaptados ao ambiente marinho os placodontes e notossauros - sendo todos eles agrupados em Sauropterygia (Fig. 3). Muitos autores (ver Laurin \& Gauthier 2000) sustentam que os sauropterígios se originaram a partir de um ancestral comum compartilhado com as serpentes e lagartos - formando o grupo dos lepidossauromorfos (grupo dos répteis com escama). Algumas 

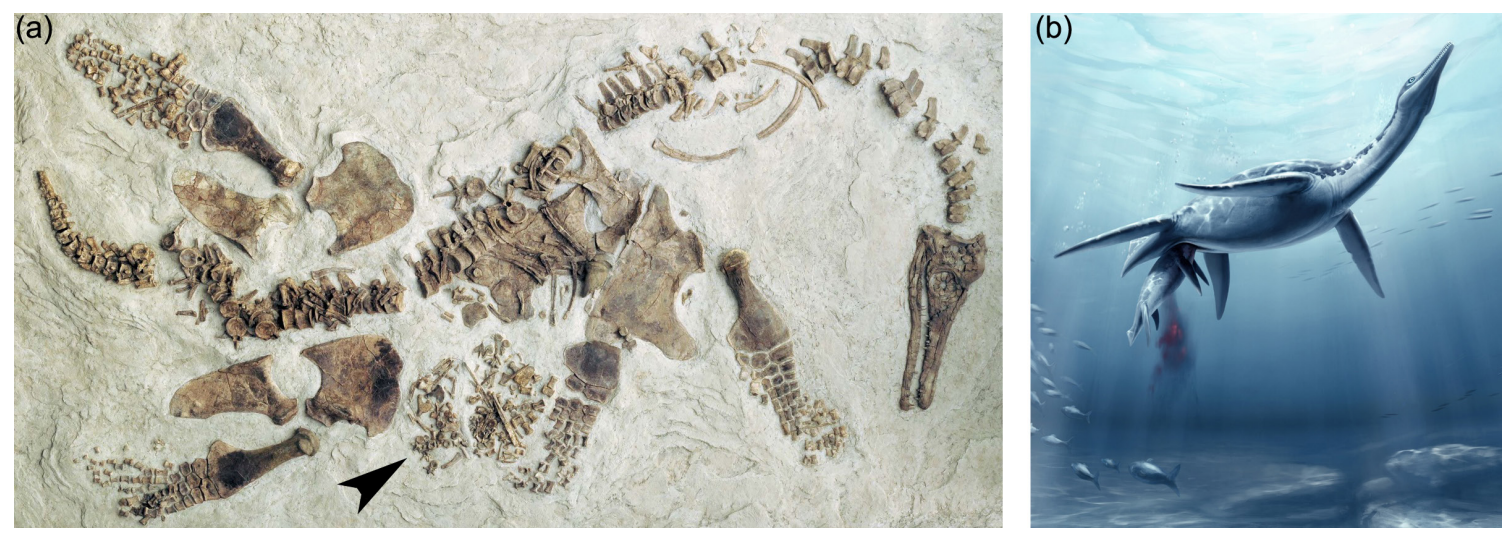

Figura 5. Plesiossauro do gênero Polycotylus. (a) Fóssil com cerca de 4 m de comprimento, preservando restos do embrião (seta). (b) Representação artística do nascimento de um plesiossauro. Imagens: (a) O'Keefe \& Chiappe (2011), (C) The American Association for the Advancement of Science; (b) Stephanie Abramowicz, Dinosaur Institute of the Natural History Museum of Los Angeles County.

propostas mais antigas colocavam os plesiossauros como parentes mais próximos dos ictiossauros (ver Caldwell 1999), e que estes dois grupos formavam uma única subclasse chamada Euryapsida - devido à presença de aberturas bem peculiares na parte posterior do crânio. A hipótese fez com que os ictiossauros e plesiossauros ficassem conhecidos como dragões-do-mar (Hawkins 1840, Benton 2006). No entanto, estudos mais recentes, como a descoberta do Cartorhynchus, por exemplo, apontam que os grupos formavam linhagens evolutivas bem separadas.

Os plesiossauros apareceram no final do período Triássico (cerca de $200 \mathrm{Ma}$ ) e se extinguiram no fim do Cretáceo (66 Ma) (Benton 2006). No entanto, pelo menos um "plesiossauro" ainda está vivo no imaginário popular. Nas Terras Altas da Escócia, a lenda de uma grande serpente habitando as águas misteriosas do Lago Ness tem espicaçado a imaginação de gerações. Pictografias e uma montagem fotográfica da década de 1930 (Fig. 6),

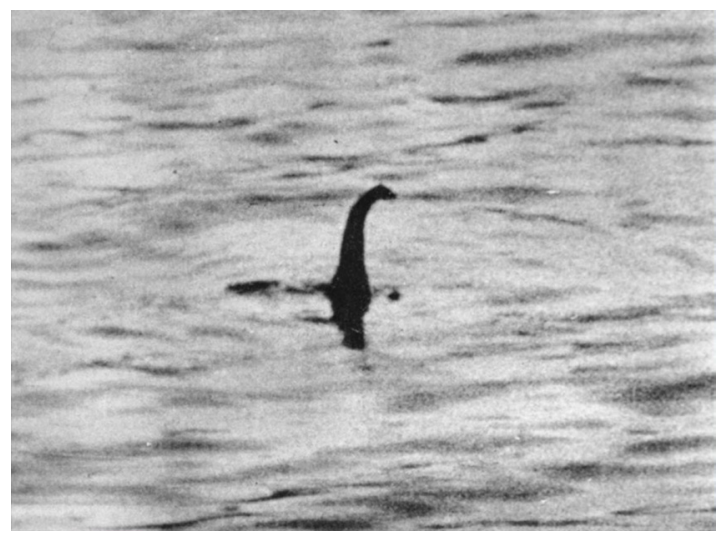

Figura 6. Imagem de 1934 "comprovando" uma aparição do monstro do Lago Ness. Na década de 1980, foi revelado que a foto era uma montagem. Imagem: Martin \& Boyd (1999). comprovando a "aparição" do monstro, mostram-no com pescoço longo, crânio curto, reptiliano e dorso arqueado (Martin \& Boyd 1999), semelhante a um plesiossauro descrito neste artigo. No ano de 2003, uma busca financiada pela rede inglesa BBC, com equipamentos de alta tecnologia, confirmou que Nessie (o nome dado ao monstro) é um mito.

\section{Mosassauros: os répteis do Rio Meuse}

Os mosassauros foram os primeiros répteis extintos a serem reconhecidos pela ciência (Evans, 2010) e por isso tiveram uma importância muito grande no desenvolvimento das ciências naturais nos séculos XVIII e XIX. Os primeiros achados ocorreram em 1764, na região de Maastricht, na Holanda (Fig. 7), e daí vem seu nome. Mosa é a forma latina de Meuse (ou Maas), que se refere ao rio que cruza essa cidade holandesa.

A descoberta desse animal foi crucial no desenvolvimento da teoria da evolução biológica. Nos primeiros anos de 1800, o naturalista francês Georges Cuvier usou o mosassauro como evidência da extinção de espécies ao longo do tempo um importante aspecto da teoria evolutiva que se desenvolveria mais solidamente na segunda metade do século 19.

Diferentemente dos ictiossauros e plesiossauros, os mosassauros viveram exclusivamente no Cretáceo Superior, isto é, entre 100 e 66 Ma (Polcyn et al. 2008). Como os demais répteis aquáticos apresentados acima, os mosassauros são claramente adaptados ao ambiente aquático (Fig. 8). Isso pode ser atestado pela forma alongada do corpo, narina externa no topo do crânio, membros modificados em nadadeiras, hiperfalangia e cauda natatória (Car- 


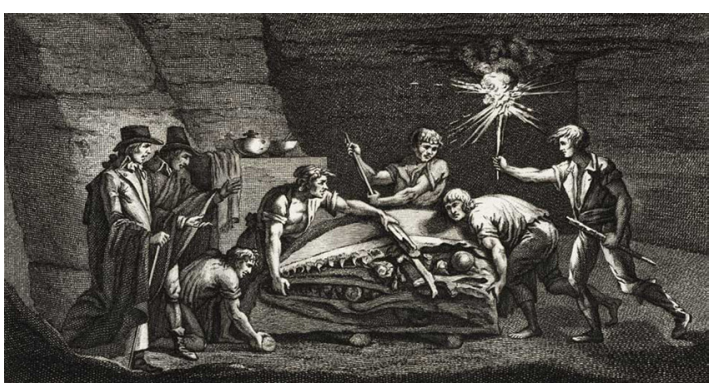

Figura 7. Gravura do século XVIII mostrando a descoberta do crânio do mosassauro. O crânio está em escala de tamanho maior que a real. Imagem: FaujasSaint-Fond (1798).

roll 1988, Pough et al. 1999, Benton 2006). Apesar de o nome ser associado ao rio Meuse (o local geográfico onde os fósseis foram encontrados), a grande maioria dos mosassauros vivia em ambiente marinho (ver Makádi et al. 2012).

O crânio da maioria dos mosassauros era alongado, especialmente na parte frontal (Carroll 1988, Pough et al. 1999). Os dentes eram maciços e fortemente associados aos ossos alveolares por ligamentos periodontais, feixes de fibras de colágeno e uma espessa cimentação celular (Caldwell et al. 2003). Os ossos da mandíbula formavam uma junção móvel adicional, permitindo maior força na mordida e captura da presa (LeBlanc et al. 2013). Sua alimentação incluía peixes, outros vertebrados (Everhart 2004) e, possivelmente, amonitas - moluscos cefalópodes extintos com concha espiralada (Hewitt e Westermann 1990, Kase et al. 1998).

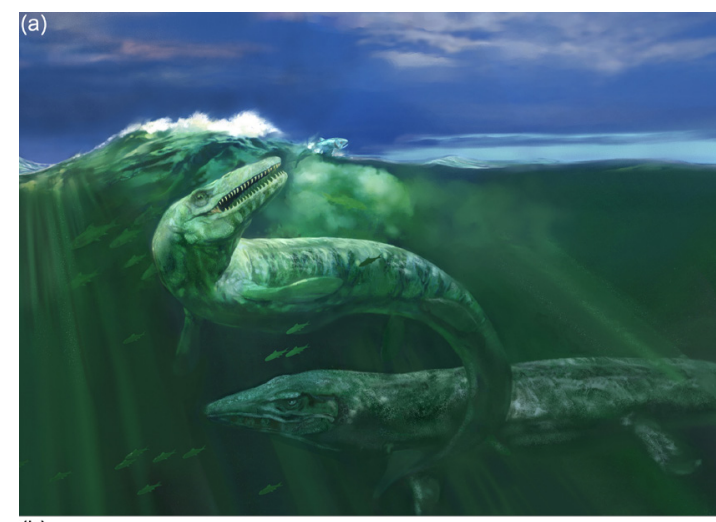

(b)

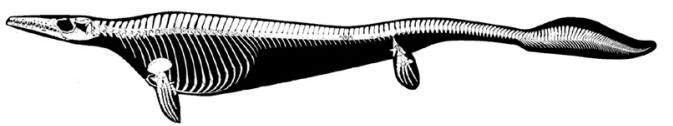

Figura 8. Exemplos de mosassauros. (a) 0 gênero que dá nome ao grupo, Mosasaurus (c. $15 \mathrm{~m}$ ). (b) Reconstrução do esqueleto de um Plotosaurus (12 m). Imagens: (a) Karen Carr Studios; (b) Motani (2009)
O nado era basicamente produzido por movimentação lateral das partes mais posteriores do corpo: a cauda era longa e anatomicamente peculiar, sendo curvada para baixo e mais expandida na parte ventral, com um lobo dorsal fazendo com que esta se assemelhasse às dos tubarões (Lindgren et al. 2011). Fósseis de mosassauros com vértebras caudais ossificadas umas às outras sugerem a ocorrência de patologias que também afetam as baleias atuais, devido ao estresse de movimentação (Rothschild e Everhart 2015). A pele destes animais tinha pigmentação (Lindgren et al. 2014) e seria recoberta por escamas reforçadas com osso dérmico, conferindo resistência e hidrodinâmica (Lindgren et al. 2009).

Os mosassauros podiam variar de 1 a 15 metros de comprimento, dependendo da espécie. Eram predadores ativos que habitavam a zona pelágica dos oceanos, ou seja, mais próximo à superfície. Alguns fósseis também mostram evidência de viviparidade (Field et al. 2015), como nos ictiossauros e plesiossauros.

As relações de parentesco dos mosassauros são mais bem esclarecidas do que as dos ictiossauros e plesiossauros. A maioria dos pesquisadores acredita que estes animais evoluíram de répteis terrestres do grupo dos escamados (Fig. 3), que inclui as serpentes e lagartos atuais (Benton 2006). A filogenia dos escamados, no entanto, é muito controversa, e os mosassauros podem ter parentesco evolutivo mais próximo com os varanoides, um grupo de lagartos que inclui o dragão-de-komodo e os lagartos-monitores, ou com as serpentes (Reeder et al. 2015).

\section{Outros grupos}

Ictiossauros, plesiossauros, mosassauros e seus parentes mais próximos foram os mais abundantes, mas não os únicos répteis que floresceram nos mares da Era Mesozoica (Fig. 3, Pough et al. 1999, Motani 2009). Outros grupos podem ser citados, como as serpentes marinhas do Cretáceo, incluindo algumas formas com membros posteriores (Caldwell \& Lee 1997; Benton 2006); quelônios, sendo um dos fósseis mais antigos de tartaruga marinha, Santanachelys, encontrado em rochas da Bacia do Araripe, no nordeste do Brasil (Hirayama 1998); e os talatossúquios, um grupo de crocodilomorfos - táxon que inclui os jacarés atuais (Riff et al. 2012) - que existiu no final do Jurássico e início do Cretáceo (Pough et al. 1999, Benton 2006).

Um grupo adicional de répteis marinhos restri- 
tos ao período Triássico são conhecidos como talatossauros (Motani 2009). É um grupo enigmático, já que não há consenso sobre com que grupo de répteis eles são mais aparentados, isto é, se representam uma linhagem filogeneticamente próxima dos ictiossauros; dos lagartos e serpentes; dos crocodilos e aves, ou se são um grupo mais primitivo de répteis, separado dessas linhagens (Liu e Rieppel 2005). Talatossauros e talatossúquios possuíam diversas convergências anatômicas (características semelhantes não por parentesco, mas por adaptação) com os répteis marinhos descritos neste artigo, devido às adaptações comuns ao ambiente aquático.

\section{Conclusões}

Durante a Era Mesozoica, os répteis foram muito diversos e abundantes em quase todos os ambientes: os continentes, a atmosfera, oceanos e mares. Porém, ao final do Mesozoico, a Terra sofreu um dos maiores eventos de extinção jamais registrados. Uma série de fatores está associada a essa perda de diversidade: intensas erupções vulcânicas, que elevaram a quantidade de gases do efeito estufa e material particulado na atmosfera; fragmentação de hábitats, ocasionada por um evento de regressão marinha (diminuição do nível do mar) no final do Cretáceo, e, possivelmente, o choque de um corpo celeste há cerca de 65 $\mathrm{Ma}$, que teria desencadeado um cataclismo global - incêndios, tsunamis, acumulação de material na atmosfera diminuindo a produtividade primária dos ecossistemas. O fim do Cretáceo e início do Paleoceno também foi marcado por um evento global de resfriamento, que provavelmente afetou toda a biota da época (Archibald \& Fastovsky 2004, Brusatte et al. 2015). No entanto, a explicação não pode ser generalizada para todos os grupos, já que os ictiossauros se extinguiram pelo menos 30 milhões de anos antes (aproximadamente quando os mosassauros se diversificaram), e os plesiossauros já estavam em franco declínio em termos de diversidade e abundância no final do Cretáceo (Fig. 3).

Os efeitos da extinção do fim do Cretáceo foram tão dramáticos para a Terra e a vida presente, que os geólogos encerraram a Era Mesozoica e denominaram o tempo subsequente de Era Cenozoica (vida recente). A partir dessa época, houve amenização do clima no interior de vastas porções dos continentes, até então árido com pouco gelo nas calotas polares e níveis marinhos altos. A amenização foi proporcionada pelos efeitos da umidade advinda da instalação plena dos oceanos Atlântico Norte (consequência da efetiva separação entres as placas tectônicas da América do Norte e Euroasiática), e o Atlântico Sul (consequência da separação das placas da América do Sul e África). Mudanças ambientais, aliadas à explosão generalizada da vida vegetal e o desaparecimento dos grandes répteis, constituíam, para as espécies, um verdadeiro mundo novo.

Pterossauros, ictiossauros, plesiossauros, mosassauros e a maior parte dos dinossauros do Mesozoico despareceram sem deixar descendentes. Isso abriu caminho para a diversificação dos mamíferos, que se tornaram os componentes predominantes de grande parte dos ecossistemas. De certa forma, a evolução do homem está indiretamente ligada aos eventos catastróficos do fim do Mesozoico, mostrando como as grandes extinções são importantes para a evolução de novas formas de vida.

\section{Questão para pesquisa e trabalhos em grupo}

1. Quais as diferenças entre os grandes animais marinhos do período Cretáceo em relação aos de hoje?

2. Além da extinção dos dinossauros, que outros grandes eventos de extinção são conhecidos na história da Terra?

3. Por que é tão difícil reconstruir as relações de parentesco entre os organismos, especialmente dos grupos extintos?

4. O texto mostra como as novidades científicas são importantes para o avanço do conhecimento. Discuta outras descobertas que foram importantes para a história da ciência e como elas afetaram a sociedade.

\section{Agradecimentos}

Os autores agradecem aos paleoartistas que gentilmente autorizaram o uso das imagens, e aos revisores, que ajudaram a melhorar a versão final deste artigo. $\mathrm{O}$ trabalho foi produzido com apoio da FAPEMIG (APQ-00517-13).

\section{Referências}

Archibald J.D., Fastovsky D.E. 2004. Dinosaur extinction. In: Weishampel D.B., Dodson, P., Osmólska H. 2004. eds. The Dinosauria. $2^{\mathrm{a}}$ ed. Berkeley: Univ. California Press. p. 672-684. 
Bernard A., Lecuyer C., Vincent P., Amiot R., Bardet N., Buffetaut E., Cuny G., Fourel F., Martineau F., Mazin J. M., Prieur A. 2010. Regulation of body temperature by some Mesozoic marine reptiles. Science, 328:1379-1382.

Benton M.J. 2006. Paleontologia dos Vertebrados. São Paulo: Atheneu.

Böttcher R. 1990. Neue Erkenntnisse über die Fortpflanzungsbiologie der Ichthyosaurier (Reptilia). Stutt. Beitr. Naturkd., B, 164:1-51.

Brusatte S.L., Butler R.J., Barrett P.M., Carrano M.T., Evans D.C., Lloyd G.T., Mannion P.D., Norell M.A., Peppe D.J., Upchurch P., Williamson T.E. 2015. The extinction of the dinosaurs. Biol Rev, 90:628-642.

Caldwell M.W. 1999. Squamate phylogeny and the relationships of snakes and mosasauroids. Zool J Linn Soc, 125:115-147.

Caldwell M.W., Budney, L.A., Lamoureux D.O. 2003. Histology of tooth attachment tissues in the Late Cretaceous mosasaurid Platecarpus.J Vert Paleontol, 23:622-630.

Caldwell M.W., Lee M.S.Y. 1997. A snake with legs from the marine Cretaceous of the Middle East. Nature, 386:705-709.

Carroll R.L. 1988. Vertebrate paleontology and evolution. New York: W.H. Freeman.

De la Beche H.T., Conybeare W.D. 1821. XXX.Notice of the discovery of a new Fossil Animal, forming a link between the Ichthyosaurus and Crocodile, together with general remarks on the Osteology of the Ichthyosaurus. Trans Geol Soc London, Series 1, 5:559-594

Evans M. 2010. The roles played by museums, collections and collectors in the early history of reptile palaeontology. Geol Soc, London, Spec Pu$b l, \mathbf{3 4 3}: 5-29$.

Everhart M.J. 2004. Plesiosaurs as the food of mosasaurs; new data on the stomach contents of a Tylosaurus proriger (Squamata; Mosasauridae) from the Niobrara Formation of western Kansas. The Mosasaur, 7:41-46.

Field D.J., LeBlanc A., Gau A., Behlke A.D. 2015. Pelagic neonatal fossils support viviparity and precocial life history of cretaceous mosasaurs. Palaeontology, 58:401-407.

Hawkins T. 1840. The Book of the Great Sea-Dragons, Ichthyosauri and Plesiosauri, [gedolim taninim] gedolim taninim, of Moses. Extinct monsters of the ancient earth. With thirty plates, copied from skeletons in the author's collection of fossil organic remains. (Depos. in the British Museum). London: W. Pickering.

Hewitt R.A., Westermann G.E.G. 1990. Mosasaur tooth marks on the ammonite Placenticeras from the Upper Cretaceous of Alberta, Canada. Can J Earth Sci, 27:469-472.
Hirayama R. 1998. Oldest known sea turtle. Nature, 392:705-708.

Kase T., Johnston P.A., Seilacher A., Boyce J.B. 1998. Alleged mosasaur bite marks on Late Cretaceous ammonites are limpet (patellogastropod) home scars. Geology, 26:947-950.

Kear B.P., Boles W.E., Smith E.T. 2003. Unusual gut contents in a Cretaceous ichthyosaur. Proc $R$ Soc Biol Sci Ser B, 270:S206-S208.

Langer M.C., Ezcurra M.D., Bittencourt J.S., Novas F.E. 2010. The origin and early evolution of dinosaurs. Biol Rev, 85:55-110.

Laurin M., Gauthier, J. 2000. Diapsid Phylogeny. Tree of Life Web Project. URL http://tolweb.org. Acesso 01.01.2016.

LeBlanc A.R.H., Caldwell M.W., Lindgren J. 2013. Aquatic adaptation, cranial kinesis, and the skull of the mosasaurine mosasaur Plotosaurus bennisoni.J Vert Paleontol, 33:349-362.

Lindgren J., Alwmark C., Caldwell M.W., Fiorillo A.R. 2009. Skin of the Cretaceous mosasaur Plotosaurus: implications for aquatic adaptations in giant marine reptiles. Biol Lett, 5:528-531.

Lindgren J., Polcyn M.J., Young B.A. 2011. Landlubbers to leviathans: evolution of swimming in mosasaurine mosasaurs. Paleobiology, 37:445-469.

Lindgren J., Sjovall P., Carney R.M., Uvdal P., Gren J.A., Dyke G., Schultz B.P., Shawkey M.D., Barnes K.R., Polcyn M.J. 2014. Skin pigmentation provides evidence of convergent melanism in extinct marine reptiles. Nature, 506: 484-488.

Makádi L., Caldwell M.W., Osi A. 2012. The first freshwater mosasauroid (Upper Cretaceous, Hungary) and a new clade of basal mosasauroids. PLoS ONE, 7: 1-16.

Martin D.S., Boyd, A. 1999. Nessie: the Surgeon's photograph exposed. London: Thorne.

Motani R. 2009. The Evolution of Marine Reptiles. Evo Edu Outreach, 2:224-235.

Motani R. s/d. Gigantes dos Mares Jurássicos. Sci Am Brasil, Edição Especial, 5:6-13.

Motani R., Jiang D.Y., Tintori A., Rieppel O., Chen G.B. 2014. Terrestrial origin of viviparity in Mesozoic marine reptiles indicated by Early Triassic embryonic fossils. PLoS ONE, 9:1-6.

Motani R., Jiang D.Y., Chen G.B., Tintori A., Rieppel O., Ji C., Huang J.D. 2015. A basal ichthyosauriform with a short snout from the Lower Triassic of China. Nature, 517:485-488.

Motani R., Rothschild B.M., Wahl W. 1999. Large eyeballs in diving ichthyosaurs. Nature, 402:747.

Motani R., You H., McGowan C. 1996. Eel-like swimming in the earliest ichthyosaurs. Nature, 382:347-348.

Nicholls E.L., Manabe M. 2004. Giant ichthyosaurs of the Triassic - A new species of Shonisaurus from the Pardonet Formation (Norian: Late Triassic) 
of British Columbia. J Vert Paleontol, 24:838-849.

O'Keefe F.R. 2001. Ecomorphology of plesiosaur flipper geometry. J Evol Biol, 14:987-991.

O'Keefe F.R. 2002. The evolution of plesiosaur and pliosaur morphotypes in the Plesiosauria (Reptilia: Sauropterygia). Paleobiology, 28:101-112.

O'Keefe F.R., Chiappe L.M. 2011. Viviparity and K-Selected Life History in a Mesozoic Marine Plesiosaur (Reptilia, Sauropterygia). Science, 333:870-873.

Organ C.L., Janes D.E., Meade A., Pagel M. 2009. Genotypic sex determination enabled adaptive radiations of extinct marine reptiles. Nature, 461:389-392.

Psihoyos L. 1994. Hunting dinosaurs. New York: Random House.

Pough F.H., Heiser J.B., McFarland W. 1999. A Vida dos Vertebrados. São Paulo: Atheneu.

Reeder T.W., Townsend T.M., Mulcahy D.G., Noonan B.P., Wood P.L., Sites J.W., Wiens J.J. 2015. Integrated analyses resolve conflicts over squamate reptile phylogeny and reveal unexpected placements for fossil taxa. Plos One, 10:1-22.

Riff D., de Souza R.G., Cidade G.M., Martinelli A.G., Souza-Filho, J.P. 2012. Crocodilomorfos: a maior diversidade de répteis fósseis do Brasil. Terrae Didatica, 9:12-40.

Rothschild B., Everhart M.J. 2015. Co-Ossification of vertebrae in mosasaurs (Squamata, Mosasau- ridae); evidence of habitat interactions and susceptibility to bone disease. Trans. Kansas Acad. Sci., 118:265-275.

Sachs S. 2005. Redescription of Elasmosaurus platyurus Cope, 1868 (Plesiosauria: Elasmosauridae) from the Upper Cretaceous (Lower Campanian) of Kansas, U.S.A. Paludicola, 5:92-106.

Faujas-Saint-Fond B. 1798. Histoire Naturelle de la Montaigne de S.-Pierre de Maestricht. Paris: H. J. Jensen.

Sgarbi G.N.C. 1999. Dinossauros: extinção ou evolução? Presença Pedagógica, 27:88-98.

Sgarbi G.N.C., Morato L. 2003. Pterossauros, répteis voadores que um dia dominaram os céus. Presença Pedagógica, 50:66-75.

Schmeisser R.L., Gillette D.D. 2009. Unusual occurrence of gastroliths in a polycotylid plesiosaur from the Upper Cretaceous Tropic Shale, Southern Utah. Palaios, 24:453-459.

Torrens H. 1995. Presidential Address: Mary Anning (1799-1847) of Lyme; 'the greatest fossilist the world ever knew'. Br J Hist Sci, 28:257-284.

Turner S., Burek C.V., Moody R.T.J. 2010. Forgotten women in an extinct saurian (man's) world. Geol Soc, London, Spec Publ, 343:111-153.

Vitt L.J., Caldwell J.P. 2014. Herpetology: An Introductory Biology of Amphibians and Reptiles. $4^{\mathrm{a}} \mathrm{ed}$. San Diego: Academic Press.

RESUMO: A Era Mesozoica é um período fascinante para a evolução da vida na Terra. Diversos grupos com representantes na fauna atual, como os lissanfíbios, mamíferos, aves, tartarugas, lagartos, serpentes e crocodilos surgiram e se diversificaram nessa era. Os dinossauros e pterossauros também ocorrem nesse intervalo do tempo geológico, tornando-0 conhecido como a Era dos Répteis. Menos conhecido do grande público é o fato de que os mares e oceanos dessa época também foram dominados por répteis extintos, incluindo ictiossauros, plesiossauros e mosassauros. Sua singular anatomia e completa extinção no fim do Mesozoico exercem fascínio desde que foram descobertos a partir do século 18.0 presente trabalho tem como objetivo fornecer subsídios ao ensino de ciências, biologia e geografia para docentes da Educação Básica (Ensino Fundamental e Médio) sobre os principais grupos de répteis marinhos da Era Mesozoica, abordando anatomia, modo de vida, evolução, além de aspectos sobre sua descoberta e implicações para a história da ciência.

PALAVRAS-CHAVE: Paleontologia, extinção, répteis fósseis, paleoambientes aquáticos. 